\title{
Q1 Q2 Shear-thinning behaviour of dense, stabilised suspensions of plate-like particles. Proposed structural model.
}

\author{
Q5 Q3 J.L. Amorós ${ }^{\mathrm{a}}$, E. Blasco ${ }^{\mathrm{a}}$, V. Beltrán ${ }^{\mathrm{b}}$ \\ Q4 a Instituto de Tecnología Cerámica, Asociación de Investigación de las Industrias Cerámicas, Spain \\ 6 b Department of Chemical Engineering, Universitat Jaume I, Campus Universitario Riu Sec, 12006 Castellon, Spain
}

\section{A R T I C L E I N F O}

\section{Article history:}

Received 17 February 2014

Received in revised form 1 June 2015

Accepted 4 June 2015

Available online $\mathrm{xxxx}$

\section{Introduction}

Certain technologies used to refine and improve the industrial properties of kaolins (such as modern wet processing) and most practical applications of kaolins (such as the processing of ceramics or paper) need highly concentrated, stable kaolin dispersions with controlled rheological properties. To prepare homogeneous suspensions of kaolin with up to $70 \%(w / w)$ solids, anionic dispersants, mainly involving silicates, polyphosphates, and polyacrylates, are added (Bergaya et al., 2006).

The rheological behaviour of dense suspensions of plate-like particles is very complex, even for well-stabilised dispersions. In fact, it was verified in a previous paper (Amorós et al., 2010) that the flow curves of electrostatically well-stabilised, highly concentrated kaolin dispersions exhibit a shear-thinning segment that is sometimes followed by a shear-thickening segment. Although much theoretical and experimental work has been done, no theory currently appears fully able to predict the evolution of the flow curves with volume fraction in the case of anisotropic particles (Philippe et al., 2013). For colloidal hard spheres, the first and one of the most appropriate ways to calculate the effective hard sphere diameter was the Barker-Henderson model, based on the perturbation theory for fluids, developed in the 1960s (Barker and Henderson, 1967) (Barker and Henderson, 1976). Subsequently, various authors (Russel and Gast, 1986) (Krieger, 1972) (Beenakker, 1984) (De Kruif et al., 1985) have consequently advocated the use of effective approaches to link suspension viscosity and volume

E-mail address: encarna.blasco@itc.uji.es (E. Blasco). fraction. In that context, in previous papers (Amorós et al., 2010) 53 (Amorós et al., 2012), the rheological properties of well-stabilised dis- 54 persions of kaolin were interpreted by considering the thickness of the 55 plate-like particle with its ionic double layer as an effective thickness. 56 The effective volume fraction of the dispersions, calculated from the 57 ionic strength of the resulting solutions and the average thickness of 58 the clay mineral particles, described well the combined effect of the 59 solids volume fraction and the dispersant additions on dispersion rheo- 60 logical properties such as plastic viscosity and extrapolated yield stress, 61 both determined by applying the Bingham model, or the storage modu- 62 lus and the loss (or damping) factor, both determined in a linear visco- 63 elastic regime.

Some authors (Philippe et al., 2013) (Michot et al., 2009) (Paineau 65 et al., 2011) (Bihannic et al., 2010), adapting Quemada's equation 66 (Quemada, 1977) (Quemada, 1998) for hard spheres to the case of 67 disk-like particles (natural swelling clay minerals), were able to ratio- 68 nalise the evolution with size and volume fraction of viscosity, at 69 different shear stresses. In this approach, the effective volume fraction 70 accounts for the fluid volume trapped by the particles through their 71 average motion, which depends on the volume fraction of spheres 72 with excluded volume encompassing the particle, and an orientation 73 parameter, which depends on shear stress. In fact, as shear stress in- 74 creases, the confinement of the particles along the velocity streamlines 75 also increases, "shrinking" the effective volume of the particles. This in- 76 terpretation is in agreement with the strong shear-thinning behaviour 77 of dense, well-stabilised suspensions of plate-like particles.

The structural model developed in the present paper is based on an 79 idealised structure in which the particles form more or less compact 80 
layers, oriented to the flow. The variable selected to characterise the structure of the dispersion is the ratio of the average interlayer distance to the effective thickness of the plate-like particles. This dimensionless variable, which determines the relative viscosity of the suspension, can be related to the effective volume fraction (used in previous papers (Amorós et al., 2010; Amorós et al., 2012)) and layer compactness by geometrical arguments. The effect of shear stress on relative viscosity, in this model, is quantified by the evolution of layer compactness with shear stress. Thus, when the shear stress increases, the plate-like particles become more oriented in the flow direction, increasing layer compactness and the dimensionless interlayer distance.

In order to test the proposed model, a kaolin was selected and characterised. The kaolin was then used to prepare more than 40 aqueous suspensions, modifying the solids volume fraction between $\phi=0.20$ and $\phi=0.475$ and the dispersant (sodium silicate) content between $X_{s}=0.075$ and $X_{s}=0.225 \mathrm{mg}$ dispersant $/ \mathrm{m}^{2}$ solid. The flow curves of all suspensions were determined in the quasi-steady state.

\section{Development of the structural model to obtain the relationship between the flow curves, $\phi$, and $X_{S}$, for dense, stabilised dispersions}

2.1. Relationship between suspension viscosity $(\eta), \phi$ and $X_{S}$ at constant shear stress $(\sigma)$

A structural model was used, based on the following assumptions:

i) At high shear rates, $\dot{\gamma}$, it may be assumed, in a first approach, that the plate-like particles are oriented parallel to the flow, forming compact layers (Fig. 1). For this structure:

$\frac{\mathrm{h}}{\mathrm{e}}=\frac{\mathrm{V}_{\text {total }}-\mathrm{V}_{\text {layer }}^{\mathrm{p}}}{\mathrm{V}_{\text {layer }}^{\mathrm{p}}}=\frac{\phi_{\text {layer }}^{\mathrm{p}}-\phi}{\phi}$

where $\mathrm{V}_{\text {total }}$ and $\mathrm{VP}_{\text {ayer }}$ are the volumes of the suspension and the particle layer, $h$ is the interlayer distance and $\phi$ and $\phi$ Payer are the solids volume fractions of the suspension and the layer, respectively.

ii) It is assumed that the kaolin particles behave as thin disks, of identical thickness, "e", with diameters that display a wide distribution (Fig. 1), as a result of which the compactness of the ordered layer, $\phi_{\max }^{\mathrm{p}}=\phi_{\text {layer, }}^{\mathrm{p}}$, can reach a value of 0.9 (Qazi et al., 2010). The ratio of the average inter-particle separation, "h", to thickness, "e", or the dimensionless average distance, $h^{*}$, then becomes:

$$
h *=\frac{h}{e}=\frac{\phi_{\max }^{p}-\phi}{\phi} .
$$

iii) In view of the pronounced effect of the inter-particle dimensionless average separation distance, $\mathrm{h}^{*}$, on suspension viscosity 118 (analogous to that of $\phi$ on $\eta$ ) (Amorós et al., 2010) (Quemada, 119 1998) (Amorós et al., 2002), the following expression was chosen 120 to describe this effect:

$-\frac{d \ln \eta}{d h^{*}}=-\frac{d \eta}{\eta d h^{*}}=B \frac{1}{\left(h^{*}\right)^{2}}$

where B is the proportional coefficient.

When infinite dilution is taken as boundary condition, i.e. when

$\phi \rightarrow 0, h^{*} \rightarrow \infty,=$

where $\mu$ is the viscosity of water.

Integrating Eq. (3) with boundary condition (4) yields:

$\eta_{R}=\frac{\eta}{\mu} \cdot \exp \left(\frac{B}{h^{*}}\right)$

This equation also obeys the divergence condition, i.e. for $\mathrm{h}^{*}=0, \eta=\infty$.

Substituting Eq. (2) into Eq. (5) then gives:

$$
\eta_{R}=\exp \left(\frac{B \cdot \phi}{\phi_{\max }^{p}-\phi}\right) .
$$

iv) If it is assumed that the orientation of the particles to the flow is not completely parallel, which is what generally occurs, in- 133 cluding at high shear stresses (Philippe et al., 2013) (Bihannic 134 et al., 2010) (Fig. 2), using the same geometric arguments as in 135 i), one obtains:

$\frac{h}{c}=\frac{\phi_{\text {layer }}-\phi}{\phi}$

where "c" is the layer thickness, which is always greater than 138 "e", the particle thickness, and $\phi_{\text {layer }}$ is its volume fraction, which is always smaller than $\phi_{\max }^{p}$, corresponding to the com- 139 pact layer.

Thus, by geometry, the following is obeyed:

$\frac{c}{e}=\frac{\phi_{\max }^{p}}{\phi_{\text {layer }}}>1$

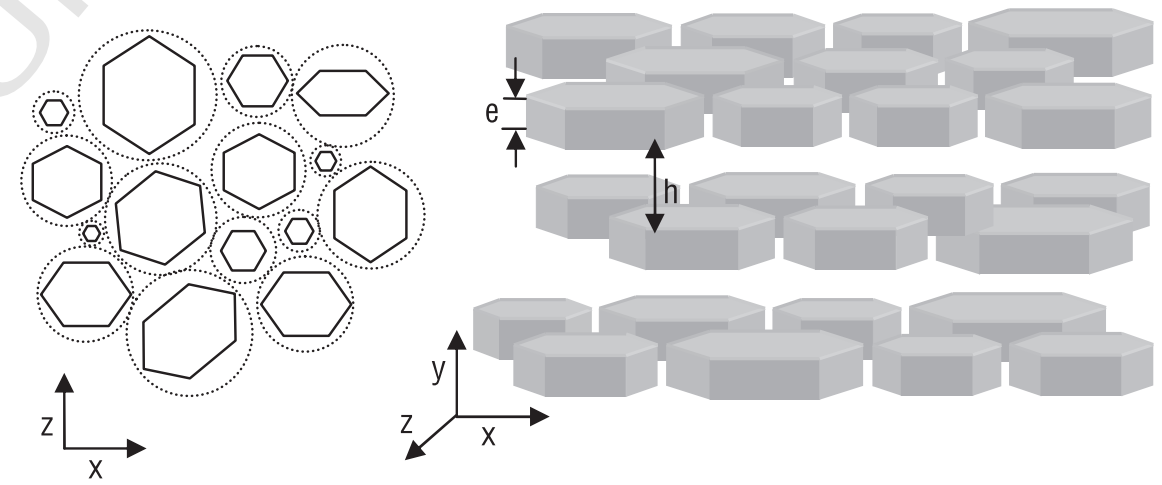

Fig. 1. Idealised structure of the kaolin suspension. Particle layers and particles entirely oriented to the flow. Flow is in the x-direction. 


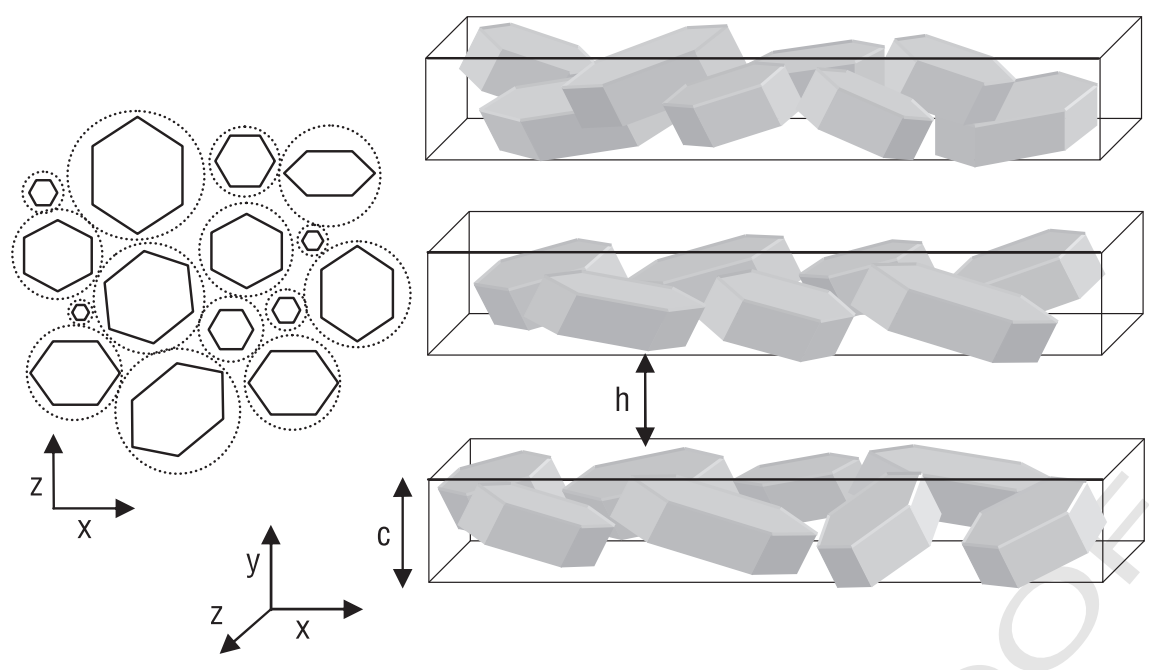

Fig. 2. Idealised structure of the kaolin suspension. Particle layers entirely, and particles partly, oriented to the flow. Flow is in the $\mathrm{x}$-direction.

$$
h^{*}=\frac{h}{e}=\frac{\phi_{\max }^{p}}{\phi_{\text {layer }}} \cdot\left(\frac{\phi_{\text {layer }}-\phi}{\phi}\right) .
$$

Introducing Eq. (9) in Eq. (5), and integrating using the infinite dilution boundary condition, one obtains:

$\eta_{R}=\exp \left(C \cdot \frac{\phi}{1-\phi / \phi_{\text {layer }}}\right)$

where $C=B / \phi_{\max }^{p}$.

v) The effect of the dispersant addition, $\mathrm{X}_{\mathrm{S}}$, on $\eta$ can be suitably described using an effective volume fraction, $\phi_{\text {eff, }}$, which only depends on the range of (repulsive) electrostatic interaction forces and which, therefore, regulates the minimum interparticle separation distance, as was assumed in a previous study (Amorós et al., 2010). For this purpose, the kaolinite particles are assumed to be hard disks dispersed in a fluid, which maintain their diameter, while their thickness, "e", increases from "e" to "e" plus twice the Debye length, $2 \cdot \kappa^{-1}$. The effective volume fraction then becomes:

$\phi_{\text {eff }}=\left(1+\frac{2 \cdot \kappa^{-1}}{e}\right) \cdot \phi$.

When the value of $\phi$ is replaced with that of $\phi_{\text {eff, }}$ and $\phi_{\text {layer }}$ is assumed to be a new value of $\phi_{e f f}^{\text {layer }}$, Eq. (10) becomes:

$$
\eta_{R}=\exp \left(C \cdot \frac{\phi_{\text {eff }}}{1-\phi_{\text {eff }} / \phi_{\text {eff }}^{\text {layer }}}\right) .
$$

\subsection{Relationship between $\phi_{\text {eff }}^{\text {layer }}$ and shear stress $(\sigma)$}

As the shear rate, $\dot{\gamma}$, or shear stress, $\sigma$, increases in any suspension, the particles become more aligned with the flow, alignment maximising at high shear, corresponding to the minimum viscosity of the suspension. This increase in the degree of particle alignment with the flow translates into increased layer compactness, $\phi_{\text {eff }}^{\text {layer }}(\sigma)$ (Fig. 3). Consequently, a dimensionless parameter can be defined that describes the effect of shear stress, $\sigma$, on the increase in layer compactness, $\phi_{e f f}^{\text {layer }}(\sigma)$, the behaviour of this parameter being similar to that of the orientation order parameter used by Egres (Egres and Wagner, 2005), or other nor- 172 malised parameters used by Jogun (Jogun and Zukoski, 1999) (elastic 173 modulus or conductivity), to study the change of particle alignment in 174 plate-like particles (kaolin).

A degree of layer compaction, $\varphi(\sigma)$, is thus defined as:

$\varphi(\sigma)=\frac{\phi_{e f f}^{\text {layer }}(\sigma)-\phi_{e f f}^{\text {layer }}(0)}{\phi_{e f f}^{\text {layer }}(\infty)-\phi_{e f f}^{\text {layer }}(0)}$

where $\phi_{\text {eff }}^{\text {layer }}(0)$ and $\phi_{\text {eff }}^{\text {layer }}(\infty)$ are the effective solids volume fraction of 178 the layer at low $(\sigma \rightarrow 0)$ and high $(\sigma \rightarrow \infty)$ shear stress (Fig. 3).

We have chosen to express $\varphi(\sigma)$ versus shear stress, $\sigma$, by a phe- 179 nomenological relation:

$\varphi(\sigma)=1-\exp \left[-\left(\frac{\sigma}{\sigma_{c}}\right)^{n}\right]$

where $\sigma_{c}$ and $\mathrm{n}$ are constant.

This relationship displays a sigmoidal dependence of $\varphi(\sigma)$ on shear stress, $\sigma$ (Fig. 3). In this relationship (Eq. 14), the parameter $\sigma_{c}$ is the 183 shear stress value where $\varphi(\sigma)=0.63$, and n describes the shape of 184 the curve (Fig. 3).

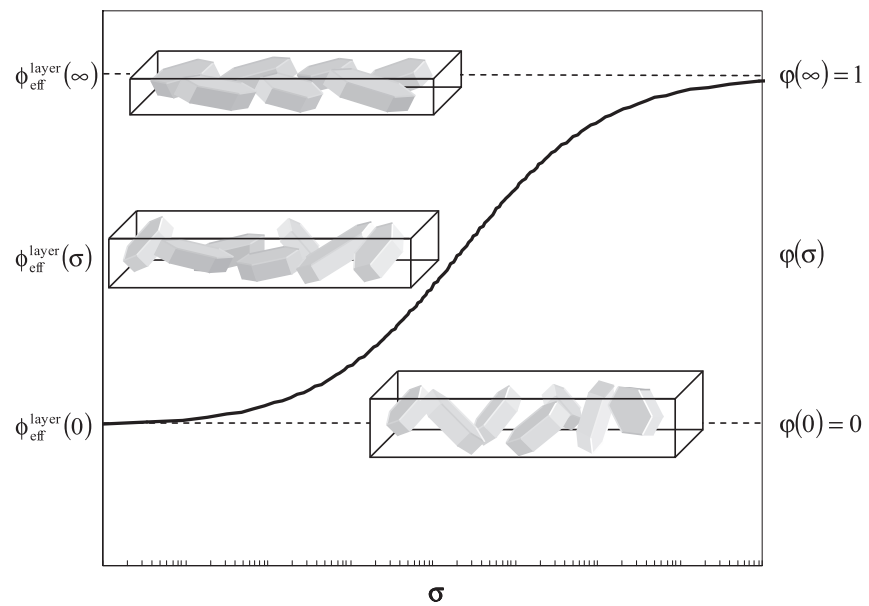

Fig. 3. Influence of shear stress, $\sigma$, on particle orientation, layer compactness, $\phi_{\mathrm{eff}}^{\text {layer }}(\sigma)$, and the degree of layer compaction, $\varphi(\sigma)$, defined by Eq. (13). 


\section{Experimental}

The proposed model was tested using a widely used commercial kaolin in the ceramic industry. The kaolin sample, referenced "F", was supplied by the firm AGS. The kaolin and its aqueous suspensions had already been physically and chemically characterised in a previous study (Amorós et al., 2010). Table 1 shows the chemical analysis of the kaolin, determined by X-ray fluorescence. The approximate mineralogical composition was: $5 \%$ quartz, $5 \%$ illite, $85 \%$ kaolinite, $1 \%$ sodium and potassium feldspar, $2 \%$ hematite and other iron and titanium compounds, and $2 \%$ other minerals. The specific surface area, determined by nitrogen adsorption (BET method) was $23.6 \pm 0.1 \mathrm{~m}^{2} / \mathrm{g}$. The mean particle size, obtained from the particle size distribution, was $410 \mathrm{~nm}$. The mean particle thickness, "e", obtained from the X-ray (001) reflection using the Scherrer equation, was $21 \mathrm{~nm}$.

A total of 42 aqueous kaolin dispersions were prepared, changing the solids volume fraction, $\phi$, from 0.20 to 0.47 and the deflocculant content, $\mathrm{X}_{\mathrm{s}}$, from 0.0705 to $0.225 \mathrm{mg}$ dispersant $/ \mathrm{m}^{2}$ clay mineral surface area. The flow curves were determined in the quasi-steady state (Amorós et al., 2002); each flow curve required 40 pairs of shear rateshear stress values. The secondary flow that affects these curves at high shears was corrected (Maranzano and Wagner, 2001).

In a previous study (Amorós et al., 2010) it was verified that the double layer thickness, $\kappa^{-1}$, was related to the solids volume fraction, $\phi$, and to the deflocculant content, $\mathrm{X}_{\mathrm{s}}$, expressed in $\mathrm{mg}$ dispersant $/ \mathrm{m}^{2}$, by means of the equation:

$\left(\kappa^{-1}\right)=\frac{0.68}{\sqrt{X s}} \cdot \sqrt{\frac{1-\phi}{\phi}}$

For the studied concentrated dispersions, $\kappa^{-1}$ changed from $5 \mathrm{~nm}$ to $1.5 \mathrm{~nm}$.

\section{Results and discussion}

\subsection{Steady-state flow curves}

The flow curves, $\eta=\eta(\sigma)$, corresponding to three of the series of suspensions, each prepared with a different dispersant content, $\mathrm{X}_{\mathrm{S}}$, are plotted as data points (squares, circles, triangles, and diamonds) in Fig. 4. The solids volume fraction $(\phi)$ was modified, in each series, in the range 0.2 to 0.485 .

It was verified that, independently of the value of $\mathrm{X}_{\mathrm{S}}$, the flow curves of all suspensions with $0.365 \leq \phi \leq 0.410$ exhibited a single shearthinning segment. In contrast, at values of $\phi \geq 0.410$ the behaviour of the suspension at high shears depended on the value of $X_{s}$. Thus, at $\mathrm{X}_{\mathrm{S}}=0.225 \mathrm{mg}$ dispersant $/ \mathrm{m}^{2}$ and high shears, the suspension was shear thickening, whereas at lower values of $X_{S}$ and volume fractions above a certain value $\left(\phi \geq 0.46\right.$ at $X_{S}=0.135 \mathrm{mg}$ dispersant $/ \mathrm{m}^{2}$ and $\phi=0.485$ at $X_{S}=0.165 \mathrm{mg}$ dispersant $/ \mathrm{m}^{2}$ ), at high shears, the curve displayed a second shear-thinning segment. The shape of the flow curve resembled that of the well-known "three-region flow curve" observed in very concentrated kaolin suspensions (Moan et al., 2003). The suspensions with $\phi=0.20$, independently of the value of $X_{S}$, were always near Newtonian.

These results confirmed the shear-thinning behaviour of electrostatically stabilised, concentrated kaolin suspensions described in the literature (Jogun and Zukoski, 1999) (Jogun, 1995) (Jogun and Zukoski,

Table 1

Chemical analysis of clay (wt.\%).

\begin{tabular}{lllllllll}
\hline $\mathrm{SiO}_{2}$ & $\mathrm{Al}_{2} \mathrm{O}_{3}$ & $\mathrm{Fe}_{2} \mathrm{O}_{3}$ & $\mathrm{TiO}_{2}$ & $\mathrm{CaO}$ & $\mathrm{MgO}$ & $\mathrm{Na}_{2} \mathrm{O}$ & $\mathrm{K}_{2} \mathrm{O}$ & LOI $^{\mathrm{a}}$ \\
\hline 47.30 & 36.40 & 1.36 & 1.13 & 0.12 & 0.17 & 0.05 & 0.85 & 12.50 \\
\hline
\end{tabular}

a Loss on ignition at $1000^{\circ} \mathrm{C}$.

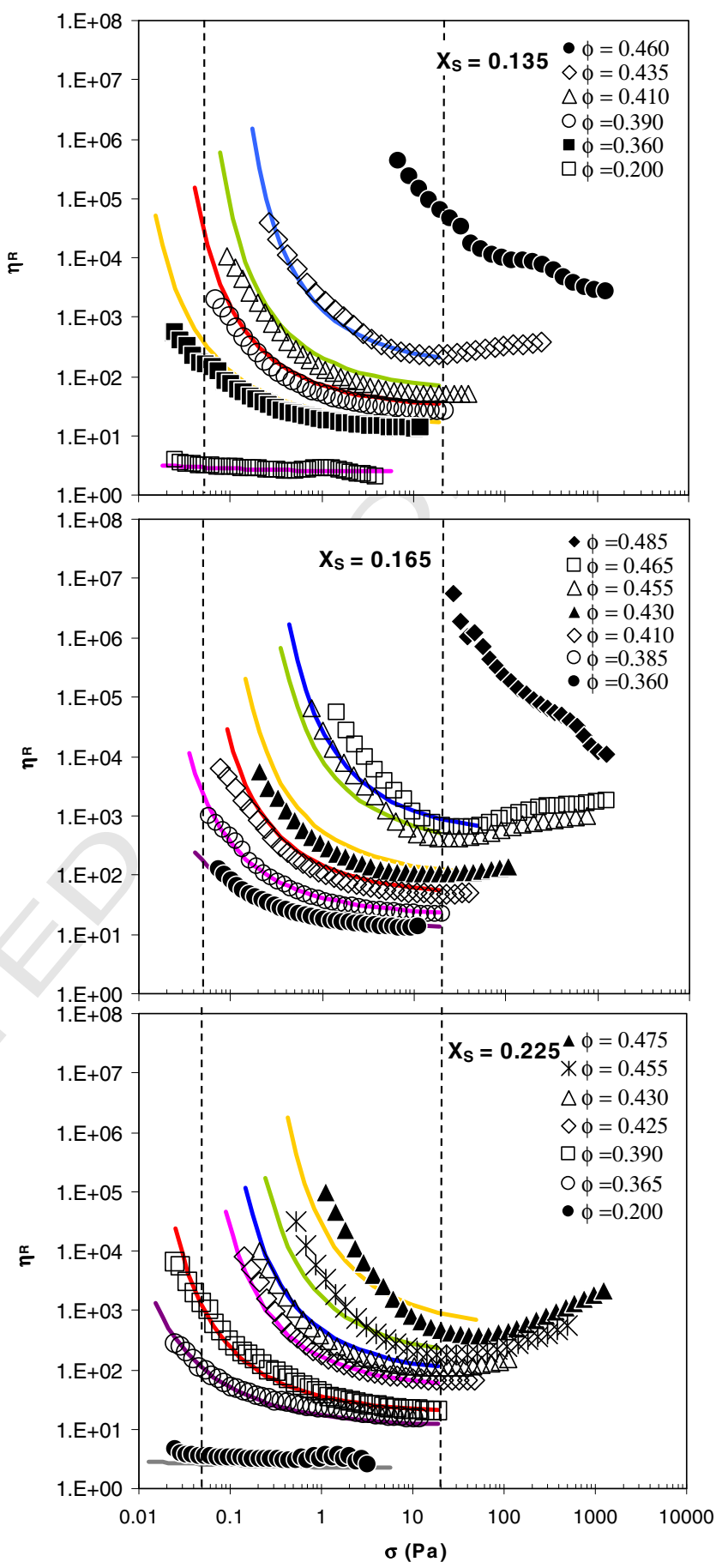

Fig. 4. Flow curves, $\eta \mathrm{R}=\eta \mathrm{R}(\sigma)$. Fit of the data (solid lines) to the model (flow diagram in Fig. 7).

1996). This behaviour is interpreted on the basis of the interaction be- 237 tween the apparent or effective volumes (exclusion effect) in concen- 238 trated suspensions of anisotropic particles such as kaolin suspensions 239 (Jogun and Zukoski, 1999) (Jogun and Zukoski, 1996) (Moan et al., 240 2003) (Bossard et al., 2007). Thus, as shear ( $\dot{\gamma}$ and $\sigma$ ) increases, the 241 plate-like particles also increasingly align in the flow direction, reducing 242 particle apparent volume, which ultimately translates into a decrease in 243 suspension viscosity.

244

With regard to shear-thickening behaviour at high shears, the effect 245 of solids content on the intensity of the phenomenon (positive curve 246 slope, $\eta=\eta(\sigma)$ ) and on the value at which $\sigma$ (flow curve minimum) 247 began to appear was analogous to that described in other systems 248 
(Maranzano and Wagner, 2001) (Barnes, 1989) (Boersma et al., 1990). However, the effect of deflocculant content, in particular, and of the inter-particle interaction forces, in general, on the observed shear thickening was much more complex, as shown elsewhere (Amorós et al., 2012).

\subsection{Verification of the proposed structural model}

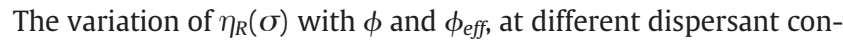
tents $\left(\mathrm{X}_{\mathrm{s}}\right)$, has been plotted, together with the fit of the results to Eq. (12), in Fig. 5. It may be observed that all values of $\eta_{R}$, which appeared scattered when they were plotted versus $\phi$, regrouped very well in a single curve, described by Eq. 12, when they were plotted against $\phi_{\text {eff }}$ (Eq. 11). The values of $\phi_{\text {eff }}^{\text {layer }}$, calculated for different values of $\sigma$, are detailed in Table 2. These values were obtained by fitting the data to Eq. 12, keeping $\mathrm{C}$ constant. The best fit was obtained by $\mathrm{C}=1.7$.
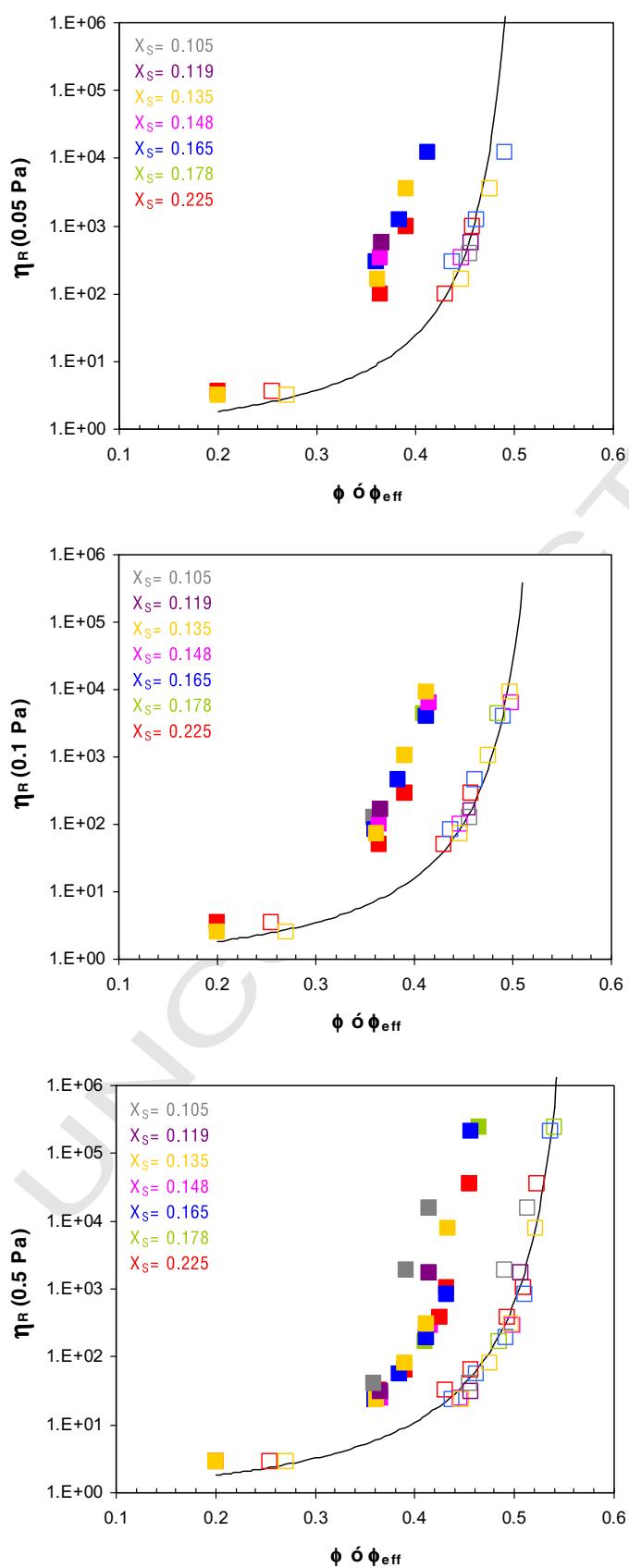

Table 2

Values of $\phi_{\text {eff }}^{\text {layer }}$ calculated by fitting the data to Eq. 12 .

\begin{tabular}{|c|c|c|}
\hline$\sigma(\mathrm{Pa})$ & C & $\phi_{\text {eff }}^{\text {layer }}$ \\
\hline 0.05 & 1.7 & 0.52 \\
\hline 0.1 & 1.7 & 0.54 \\
\hline 0.5 & 1.7 & 0.57 \\
\hline 1 & 1.7 & 0.59 \\
\hline 5 & 1.7 & 0.61 \\
\hline 10 & 1.7 & 0.62 \\
\hline 20 & 1.7 & 0.62 \\
\hline
\end{tabular}

These results confirm the validity of the developed model: on the 263 one hand, the results fit well to Eq. 12, assuming that C is independent 264 of $\sigma$ and, on the other, $\phi_{\text {eff }}^{\text {layer }}$ increases with $\sigma$ as the model predicts. 265

The values of $\phi_{\text {eff }}^{\text {layer }}$ and $\sigma$ in Table 2 were fitted using Eqs. (13) 266 and (14), which yielded a good correlation (Fig. 6). The values of 267
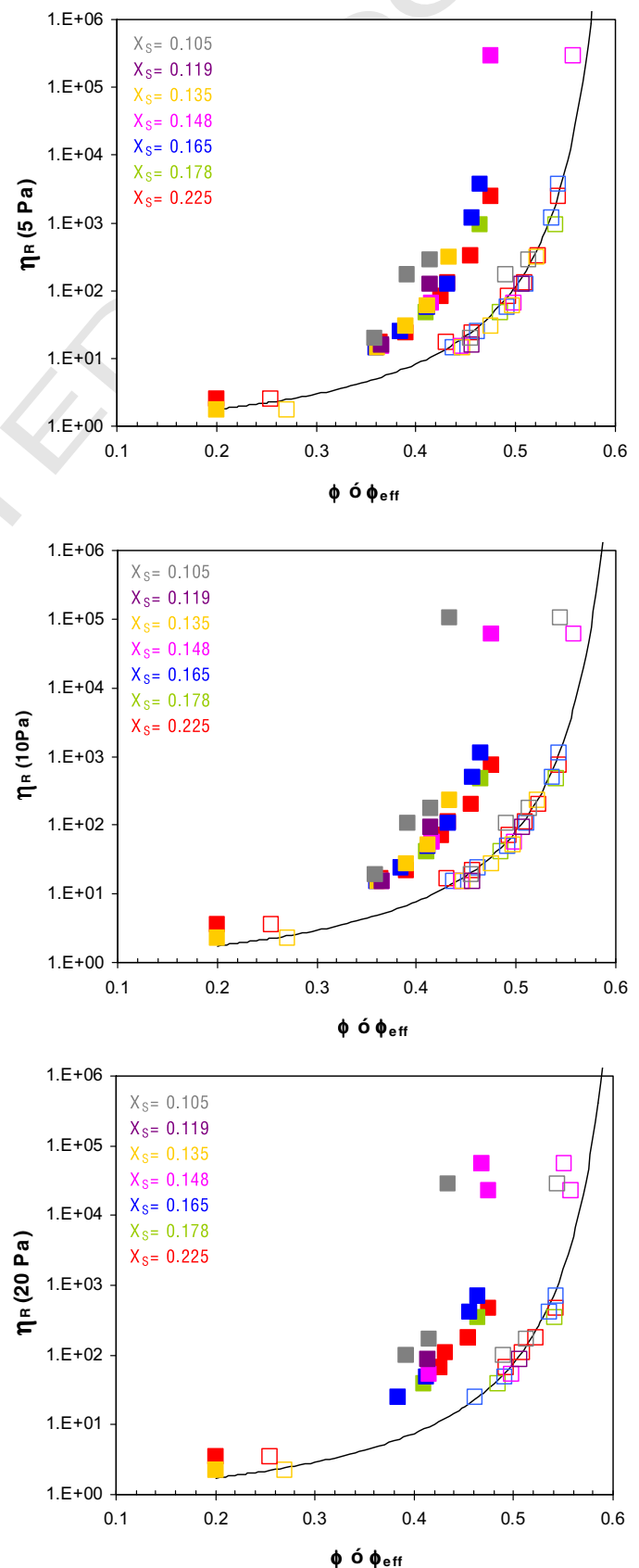

Fig. 5. Variation of $\eta \mathrm{R}(\sigma)$ with $\phi$ (filled squares) and $\phi_{\text {eff }}$ (open squares) at different dispersant contents (Xs). The solid lines represent the fit of the data to Eq. (12). 


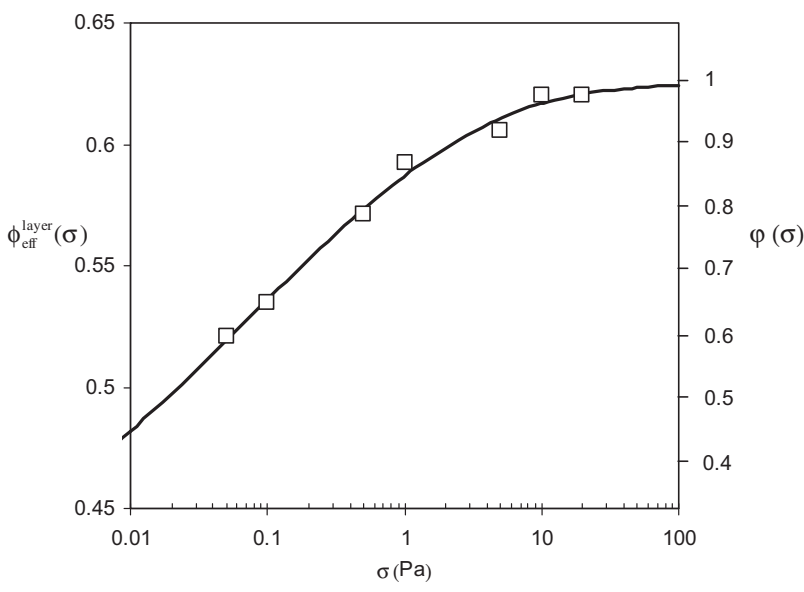

Fig. 6. Influence of shear stress, $\sigma$, on the effective layer volume fraction, $\phi_{\text {eff }}^{\text {laxer }}(\sigma)$, and the degree of layer compaction, $\varphi(\sigma)$, defined by Eq. (13).

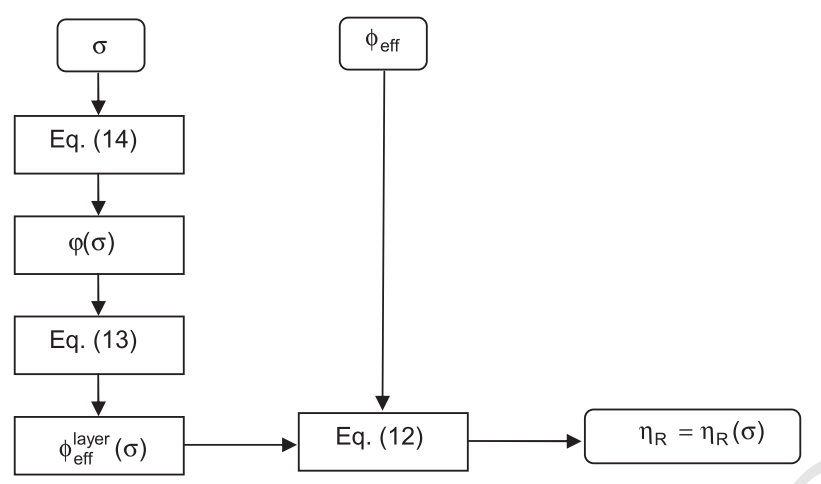

Fig. 7. Procedure followed for the reproduction of the flow curves.

The flow curves of electrostatically stabilised, concentrated kaolin 287 suspensions were determined and compared with the flow curves 288 obtained for these suspensions using the values calculated with the pro- 289 posed structural model. The results confirmed the validity of the model 290 to appropriately describe the combined effect of the solids volume frac- 291 tion and the dispersant content on the flow curves in the shear-thinning 292 range of dense, stabilised suspensions of kaolin.

\section{References}

Amorós, J.L., Sanz, V., Gozalbo, A., 2002. Viscosity of concentrated clay suspensions. Effect 295 of solids volume fraction, shear stress and deflocculant content. Br. Ceram. Trans. 101, 296 185-193.

Amorós, J.L., Beltrán, V., Sanz, V., Jarque, J.C., 2010. Electrokinetic and rheological proper- 298 ties of highly concentrated kaolin dispersions: influence of particle volume fraction 299 and dispersant concentration. Appl. Clay Sci. 49, 33-43. 300

Amorós, J.L., Blasco, E., Orts, M.J., Sanz, V., 2012. Chapter 5. Shear-thickening behaviour of 301 highly concentrated kaolin dispersions: Influence of particle volume fraction and dis- 302 persant concentration. Kaolinite: Occurrences, Characteristics and Applications, 303 pp. 117-137.

Barker, J.A., Henderson, D., 1967. Perturbation theory and equation of state for fluids II. A 305 successful theory of liquids. J. Chem. Phys. 47, 4714-4721. 306

Barker, J.A., Henderson, D., 1976. What is "liquid"? Understanding the states of matter. 307 Rev. Mod. Phys. 48, 587-671. 308

Barnes, H.A., 1989. Shear thickening ("dilatancy") in suspensions of nanoaggregating solid 309 particles dispersed in Newtonian liquids. J. Rheol. 33, 329-366. 310

Beenakker, C.W.J., 1984. The effective viscosity of a concentrated suspension of spheres 311 (and its relation to diffusion). Physica 128, 48-81. 312

Bergaya, F., Theng, B.K.G., Lagaly, G., 2006. Handbook of Clay Science. Elsevier, 313 pp. 502-506. 314

Bihannic, I., Baravian, C., Duval, J.F.L., Paineau, E., Meneau, F., Levitz, P., De Silva, J.P., 315 Davidson, P., Michot, L.J., 2010. Orientational order of colloidal disk-shaped particles 316 under shear-flow conditions: a rheological-small-angle X-ray scattering study. 317 J. Phys. Chem. B 114, 16347-16355.

Boersma, W.H., Laven, J., Stein, H.N., 1990. Shear thickening (dilatancy) in concentrated 319 dispersions. AICHE J. 36, 321-332. 320

Bossard, F., Moan, M., Aubry, T., 2007. Linear and nonlinear viscoelastic behavior of very 321 concentrated plate-like kaolin suspensions. J. Rheol. 51, 1253-1270. 322

De Kruif, C.G., Van Iersel, E.M.F., Vrij, A., Russel, W.B., 1985. Hard spheres colloidal disper- 323 sions: viscosity as a function of shear rate and volume fraction. J. Chem. Phys. 83, 324 4717-4725.

Egres, R.G., Wagner, N.J., 2005. The rheology and microstructure of acicular precipitated 326 calcium carbonate colloidal suspensions through the shear thickening transition. 327 J. Rheol. 49 (3), 719-746.

Jogun, S.M., 1995. Rheology and Microstructure of Concentrated Suspensions of Plate- 329 Shaped Colloidal Particles. B. S., Carnegie Mellon University and M. S., University of 330 Illinois, Urbana-Champaign (Thesis).

Jogun, S.M., Zukoski, C.F., 1996. Rheology of dense suspensions of platelike particles. 332 J. Rheol. 40, 1211-1232.

Jogun, S.M., Zukoski, C.F., 1999. Rheology and microstructure of dense suspensions of 334 plate-shaped colloidal particles. J. Rheol. 43, 847-871. 335

Krieger, I.M., 1972. Rheology of monodisperse latices. Adv. Colloid Interf. Sci. 3, 111-136. 336

Maranzano, B.J., Wagner, N.J., 2001. The effects of particle size on reversible shear thick- 337 ening of concentrated colloidal dispersions. J. Chem. Phys. 114, 10514-10527. 338

Michot, L.J., Baravian, C., Bihannic, I., Maddi, S., Moyne, C., Duval, J.F., Levitz, P., Davidson, 339 P., 2009. Sol/gel and isotropic/nematic transitions in aqueous suspensions of natural 340 nontronite clay. Influence of particle anisotropy. 2. Gel structure and mechanical 341 properties. Langmuir 25, 127-139.

Moan, M., Aubry, T., Bossard, F., 2003. Nonlinear behavior of very concentrated suspen- 343 sions of plate-like kaolin particles in shear flow. J. Rheol. 47, 1493-1504. 344

Paineau, E., Michot, L.J., Bihannic, I., Baravian, C., 2011. Aqueous suspensions of natural 345 swelling clay minerals. 2. Rheological characterization. Langmuir 27, 7806-7819. 346

Philippe, A.M., Baravian, C., Bezuglyy, V., Angilella, J.R., Meneau, F., Bihannic, I., Michot, L.J., 347 2013. Rheological study of two-dimensional very anisometric colloidal particle 348 suspensions: from shear-induced orientation to viscous dissipation. Langmuir 29, 349 5315-5324.

Qazi, S.J., Karlsson, G., Rennie, A.R., 2010. Dispersions of plate-like colloidal particles-cubatic 351 order? J. Colloid Interface Sci. 348, 80-84. 352

Quemada, D., 1977. Rheology of concentrated disperse systems and minimum energy dis- 353 sipation principle. Rheol. Acta 16, 82-94. 354

Quemada, D., 1998. Rheological modelling of complex fluids. I. The concept of effective 355 volume fraction revisited. Eur. Phys. J. Appl. Phys. 1, 119-127. 356

Russel, W.B., Gast, A.P., 1986. Nonequilibrium statistical mechanics of concentrated 357 colloidal dispersions: hard spheres in weak flow. J. Chem. Phys. 84, 1815-1827. 358 